\title{
Fabrication Concept for Biomimetic Microfluidic Systems
}

\author{
J. Zehetner ${ }^{1}$, S. Kasemann ${ }^{1}$, E. Kostal' ${ }^{1}$, G. Vanko ${ }^{2}$, O. Babchenko ${ }^{2}$ \\ ${ }^{1}$ Research Centre for Microtechnology, University of Applied Sciences, Hochschulstraße 1, 6850 \\ Dornbirn, Austria \\ ${ }^{2}$ Institute of Electrical Engineering, Slovak Academy of Sciences, Dubravska cesta 9, 84104 \\ Bratislava, Slovakia \\ johann.zehetner@fhv.at
}

\begin{abstract}
:
This work reports the results obtained by using a technological process combining reactive ion etching (RIE) and laser ablation in the femtosecond regime. We produce membranes, micro- and nanostructured surfaces as well as 3D structures for application oriented research. Our focus is on microfluidic devices, MEMS and pressure sensors. Primarily we use AIGaN/GaN heterostructure layers grown on $4 \mathrm{H}-\mathrm{SiC}$ or $\mathrm{Si}$ substrates, glass and ceramics. We demonstrate a novel method of direct mask writing into a thin metal layer by laser ablation for RIE compassing a micro lithography step. In order to extend the narrow ablation parameter window of our standard procedure we utilize a self-destructing etalon for a new topology selective etch mask writing process. In the subsequent RIE step we can produce up to $300 \mu \mathrm{m}$ long and in diameter $1 \mu \mathrm{m}$ to $10 \mu \mathrm{m}$ needles, $15 \mu \mathrm{m}$ hollow pillars and box like features consisting of a less than $200 \mathrm{~nm}$ thick metal casing or pure $\mathrm{Si}$. It is possible to transfer the pillars into silicon oxide and functionalize such structures by coating or filling them with gold nanoparticles or thin film semiconducting metal oxides. Some of the micro needle arrangements reveal super hydrophobic properties which makes them useful in microfluidic systems and micro bioreactors when a liquid and gas phase shall be separated to supply a gas sensor with process gas. Our previously developed membrane based pressure sensors have the potential to be fully integrated in such a system.
\end{abstract}

Key words: Pressure sensor, microfluidic, biomimetic, drumskin sensor, MEMS

\section{Introduction}

AlGaN/GaN based high electron mobility transistors (HEMT's) are used in mechanical or chemical sensors operating at extreme conditions. Motivated by the research on MEMS sensors based on III-N material systems we developed a technological method to produce membranes in AIGaN/GaN/4H-SiC substrates by combining laser ablation in the femtosecond regime and RIE [1] to reduce the backside damage on the membrane surface. Additionally challenging was the pinhole formation in the membrane during laser ablation. We realized that laser induced periodic surface structure (LIPSS) in SiC provide slot waveguides and support the growth of $2 \mu \mathrm{m}$ to $5 \mu \mathrm{m}$ wide pinholes. The pinhole growth mechanism was interrupted by steady rotation or frequent $90^{\circ}$ angular flipping of the laser radiation polarization [2]. On SiC substrates with backside metallization it was not possible to produce membranes by laser ablation. Due to an etalon effect LIPSS were formed at lower laser intensity on the SiC backside surface [2]. The understanding of such problems inspired us to develop a direct mask writing method for RIE by fs-laser ablation and an indirect variation using a self-terminating etalon. As a mask material we use mostly the metals $\mathrm{Al}, \mathrm{Ti}, \mathrm{Ni}$ and a multilayer combination metal/metal or metal/silicon-oxide. The different metals respond with a specific LIPSS pattern in sub$\mu \mathrm{m}$ dimensions and together with the laser polarization and laser scanning hatch this offers some design freedom for the generated mask structure.

\section{Experiments and Results}

For laser ablation we used a Spectra Physics SPIRIT delivering $350 \mathrm{fs}$ pulses at $200 \mathrm{kHz}$ with an average power output of $4000 \mathrm{~mW}$ at $1040 \mathrm{~nm}$ and $1600 \mathrm{~mW}$ at $520 \mathrm{~nm}$. Metal films of $\mathrm{Al}, \mathrm{Ti}$ or Ni were deposited in a PVD coating process and subsequently structured by means of laser ablation to serve as etch mask. The thickness of the metal films was $200 \mathrm{~nm}$ (in case of $\mathrm{Al}, \mathrm{Ni}$ ) and $100 \mathrm{~nm}$ (in case of $\mathrm{Ti}$ ). Deep 
Reactive lon Etching (DRIE) process was done in an adixen ${ }^{\circledR}$ AMS 100 I-Speeder system using $\mathrm{SF}_{6}$ and $\mathrm{C}_{4} \mathrm{~F}_{8}$ as precursors. We obtained a grass like appearance by direct ablation of an Al coating shown in Fig. 1. The structure is superhydrophobic exhibiting a water contact angle of $168^{\circ}$ (upper left insert). In contrast to $\mathrm{Al}$, in a $\mathrm{Ni}$ layer the laser generated stripe shaped LIPSS are perpendicular to the laser polarization. At the crossover of the two perpendicular polarized ablation tracks dots with a size of approximately $300 \mathrm{~nm}$ are formed.

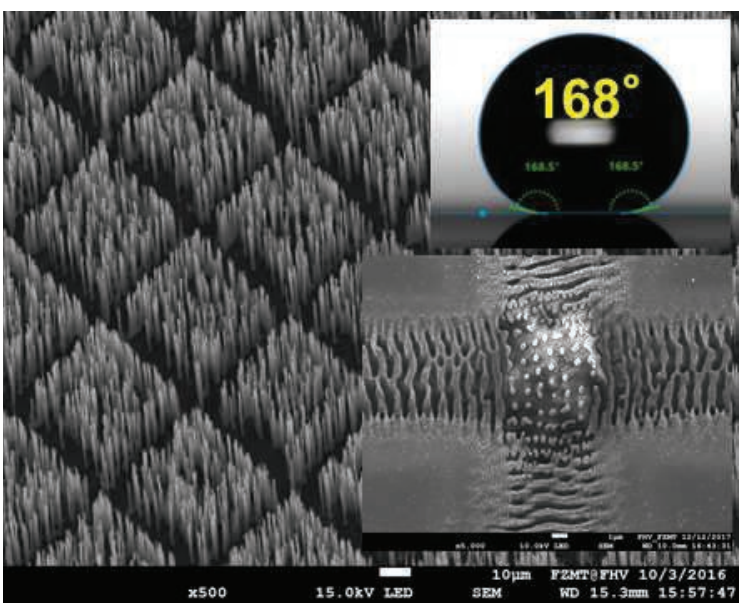

Fig. 1. Micro needles produced by DRIE using an Al-mask. The mask was produced by direct laser ablation

In zircon ceramics we produced a microfluidic channel by laser ablation with a doublehierarchical surface structure at the bottom (Fig. 2). In [3] it was demonstrated that such structures are superhydrophilc. Bonding a superhydrophobic "grass" structure on top of such a channel provides a closed channel system where liquid and gas phase can be separated and pressure sensors like in the lower insert of Fig. 2 might be integrated. Moreover we managed the fabrication of hollow micro pillars $15 \mu \mathrm{m}$ to $20 \mu \mathrm{m}$ in diameter (upper insert Fig. 2). The mask writing for such pillars as well as for the box like features in Fig. 3 was performed by using an etalon effect established between the $200 \mathrm{~nm} \mathrm{Al}$ mask coating on the Si wafer and a glass sheet coated with $50 \mathrm{~nm} \mathrm{Ti}$. The Si wafer was structured before Al coating in a square shaped $45 \times 45 \mu \mathrm{m}$ pattern of $50 \mu \mathrm{m}$ deep trenches. We tested rectangular and $\mathrm{V}$ shaped trenches. The etalon effect was dominant in the thin gap between the flat top of the squares or micro pyramid structures and selectively ablated the top Al-layer only with little influence to the side walls or pyramid slopes. The selectivity was given by the topology of the surface. After DRIE the $200 \mathrm{~nm}$ metal coating remains as a hollow metal feature. The laser parameters could be set to produce empty boxes or with inserted micro needles.

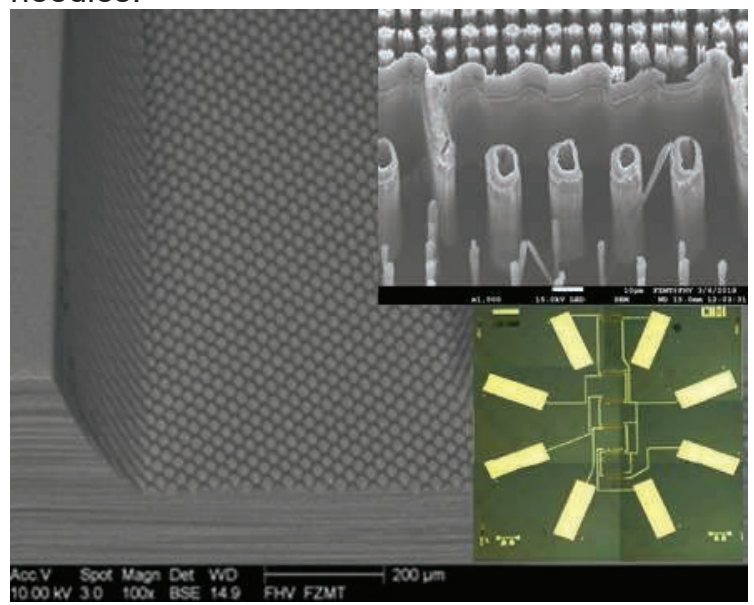

Fig. 1. Microfluidic channel produced by laser ablation. Lower insert shows a drumskin pressure sensor for direct integration in a microfluidic system



Fig. 3. Metal features consisting of a $200 \mathrm{~nm}$ Alwall fabricated by DRIE. The mask was produced by etalon supported laser ablation

\section{References}

[1] G. Vanko, P. Hudek, J. Zehetner, J. Dzuba, P. Choleva, V. Kutis, M. Vallo, I. Ryger, T. Lalinsky, Bulk micromachining of SiC substrates for MEMS sensor applications, Microelectronic Engineering 110, 260 (2013); doi: 10.1016/j.mee.2013.01.046

[2] J. Zehetner, S. Kraus, M. Lucki, G. Vanko, J. Dzuba, T. Lalinsky, Manufacturing of membranes by laser ablation in $\mathrm{SiC}$, sapphire, glass and ceramic for GaN/ferroelectric thin film MEMS and pressure sensors, Microsystem Technologies 22 (9), 1883-1892 (2016); doi: 10.1007/s00542-0162887-2

[3] E. Kostal, S. Stroj, S. Kasemann, V. Matylitsky, M. Domke, Fabrication of biomimetic fogcollecting superhydrophilic- superhydrophobic surface micropatterns using femtosecond lasers, Langmuir 34 (9), 2933-2941 (2018); doi: 10.1021/acs.langmuir.7b03699 